\title{
Ácido acetilsalicílico (AAS) inhibe angiogénesis en membrana alantocorionica de pollo (MAC)
}

\author{
Sebastián Córdova ${ }^{1,2}$, David Lemus ${ }^{1, a}$, Carlos Rosas ${ }^{1, b}$, José Luis Calderón. \\ 1. Laboratorio de Embriología Experimental y Molecular, ICBM de la Universidad de Chile \\ 2. Clínica Indisa \\ 3. Hospital San José \\ a. Médico veterinario \\ b. Tecnólogo médico

\begin{abstract}
* Esta investigación fue realizada en el laboratorio de Embriología Experimental y Molecular del programa de Anatomía y Biología del Desarrollo del Instituto de Ciencias Biomédicas (ICBM) de la Facultad de Medicina de la Universidad de Chile.
\end{abstract} \\ * Esta investigación fue desarrollada y financiada con fondos del autor.
}

Introducción: El ácido acetilsalicílico (AAS) es ampliamente utilizado en el manejo de patología cardiovascular. En modelos "in vitro" el AAS restringe la angiogénesis, atribuyéndose este efecto al bloqueo de ciclooxigenasa-1, manteniendo íntegra la zona adherente endotelial, citotoxicidad directa y otras vías de señalización.

Hipótesis: El AAS en concentración terapéutica antiplaquetaria utilizada en humanos ejerce un efecto antiangiogénico en modelo de membrana alantocoriónica de pollo (MAC).

Objetivo: Comparar la capacidad antiangiogénica del AAS en distintas concentraciones en MAC utilizando como punto de comparación la angiogénesis fisiológica de la MAC.

Método: Se incubaron 46 huevos fecundados de gallinas White Leghorn, en cámara temperada a $37^{\circ} \mathrm{C}$, provenientes del Instituto de Salud Pública de Chile. Mediante procedimiento descrito por Ribatti (2006), se instiló sobre filtro de metilcelulosa $10 \mathrm{uL}$ de Dimetilsulfóxido al $0.1 \%+\mathrm{m} 199$, sin fármaco al control, asociado a AAS y ácido salicílico (AS) a los grupos de estudio en concentraciones $2 \mathrm{mM}$ y $5 \mathrm{mM}$. Posteriormente se fijó y analizó la muestra en forma ciega. Resultados: El promedio de vasos del control fue 21.8. Para el grupo AAS $2 \mathrm{mM}$ y $5 \mathrm{mM}$ fue 11.3 y 10 , siendo para el grupo AS $2 \mathrm{mM}$ y $5 \mathrm{mM} 15.6$ y 12.4 . El análisis estadístico mediante ANOVA y t-Student muestra que todos los grupos que recibieron fármacos tuvieron una disminución significativa en el número de vasos sanguíneos en relación al grupo control. No hubo diferencias significativas entre ambo grupos de AAS. El AS demostró tener mayor potencia antiangiogénica dosis dependiente.

Discusión: En este estudio se demuestra que el AAS ejerce un efecto antiangiogénico en concentración terapéutica en condiciones fisiológicas de un modelo "in vivo".

Correspondencia:

Dr. Sebastián Ignacio Córdova Ortega.

Independencia 1027. Facultad de Medicina de la Universidad de Chile. blumberg.positivo@hotmail.com 


\section{Acetyl-salicylic acid inhibits angiogenesis in alanto chorionic chicken membrane}

Background: Acetylsalicylic acid (ASA) is widely used in the treatment of various cardiovascular disorders. In vitro, AAS decreases angiogenesis, through cyclo-oxigenase-1 blockade while keeping active the adherent endothelial zone, direct toxicity and other signaling pathways.

Hypothesis: AAS at therapeutic anti plaquetary doses exerts an anti-angiogenic effect in the alanto choronic chicken membrane (ACM)

Method: 46 fertilized eggs form White Leghorn hens were incubated at $37 \mathrm{oC} .10 \mathrm{uL}$ of $0.1 \%$ Dimethyl sulfoxide $+\mathrm{Ml} 19$ with no drug were used as control, while experimental groups received ASA and Salicylic acid (SA), 2mM. After fixation, samples were analyzed in a blind fashion

Results: The mean number of vessels was 21.8 for controls, 11.3 and 10 for ASA 2mM and ASA 5mM, respectively. Corresponding values for SA 2 and SA $5 \mathrm{mM}$ were 15.6 and 12.4 , respectively. Thus, a statistically significant (ANOVA and Student's t) decrease in the number of vessels was observed in both ASA groups. SA showed had a greater potential for anti-angiogenesis in a dose dependent way.

Conclusion: This study shows that ASA in therapeutic concentrations has an anti-angiogenic effect in a physiologic model in vivo.

Key words: Aspirin, salicylic acid, angiogenesis, chorioallantoic membrane, chicken. 
Introducción: La angiogénesis se define como el proceso en el cual toman su origen y se desarrollan vasos sanguíneos de vasos preexistentes. La vasculogénesis comprende a un proceso que ocurre en el embrión. Durante la diferenciación morfo funcional (arteriogénesis), estos contribuyen a regular la perfusión tisular asociado al recubrimiento de pericitos y células musculares. (Swift \& Weinstein., 2009; Novosel et al., 2011).

Dentro de los factores endógenos que estimulan la respuesta angiogénica se incluyen familia de factores de crecimiento vascular endotelial (VEGF), principalmente VEGF-A (Carmeliet, 2005).

Ácido acetilsalicílico (AAS) y su efecto en la angiogénesis El AAS conocido por sus efectos antiplaquetarios es utilizado en la clínica para el tratamiento y prevención de eventos cardiovasculares y además como analgésico (Toyoda., 2009). Ejerce sus efectos bloqueando a la enzima ciclooxigenasa 1 y $2(\mathrm{COX}-1$ y COX-2) y es considerado un agente antiangiogénico (Khan \& Metha., 2005).

Tanto AAS y AS comparten "in Vitro" la capacidad de mantener adheridas las proteínas de la zona adherente de las células endoteliales (Khaidakov et al., 2012). Además, AAS causa un bloqueo irreversible de la actividad plaquetaria y con ello un bloqueo de la capacidad proangiogénica plaquetaria a través del VEGF plaquetario (Battinelli et al., 2011).

La ventaja que ofrece el modelo MAC, al ser un modelo "in vivo", se debe a la actividad proangiogénica intrínseca además de las ventajas técnicas: alta tasa de supervivencia embrionaria durante el periodo de estudio, metodología estandarizada y sencilla, bajo costo, alta reproducibilidad, no requiere condiciones de esterilidad y es de alta fiabilidad. Este modelo ha sido validado en el estudio de numerosas sustancias proangiogénicas y antiangiogénicas con la misma metodología y además, se han descrito las fases de crecimiento vascular como así su porcentaje de actividad, transformándolo en un modelo predecible de crecimiento vascular, el cual ha sido clasificado en tres fases: fase de brote, que dura hasta el día 6 , fase intermedia que se mantiene hasta el día 10 (porcentaje de proliferación vascular del $50 \%$ en comparación con la fase de brote) y fase tardía hasta el día 15 en donde la actividad proliferativa desciende a menos del $10 \%$ en comparación con la fase de brote. Dentro de las desventajas, estas incluyen: dificultad para la monitorización de nuevos vasos en relación a los ya existentes y reacciones inflamatorias no especificas dependientes de cuerpo extraño (por ejemplo: filtro de instilación de metilcelulosa) (Ribatti., 2010).

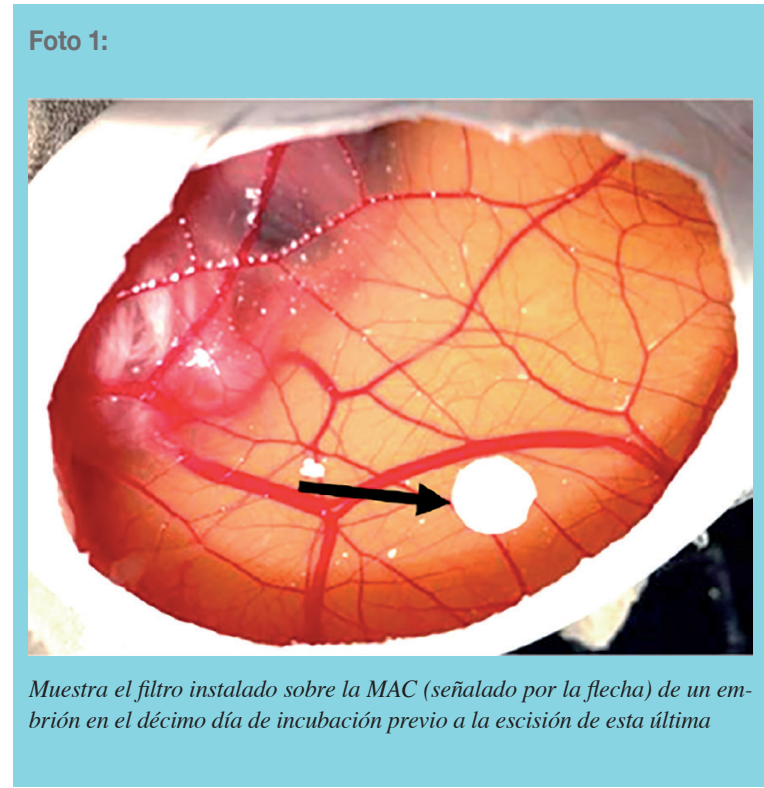

\section{Materiales y métodos}

Estudio experimental que no requirió de evaluación por comité de ética.

\section{Preparación de la MAC e instilación de fármacos:}

Se incubaron 60 huevos recién fecundados de gallinas White Leghorn procedentes del bioterio del Instituto de Salud Pública de Chile, en una cámara temperada a $37^{\circ} \mathrm{C}$ con aire humedecido, de los cuales 46 llegaron a término para ser estudiados, conformando así el grupo de estudio. AAS y AS fueron obtenidos de la Facultad de Ciencias Químicas y Farmacéuticas de la Universidad de Chile. Mediante procedimiento descrito por Ribatti (Ribatti et al., 2006), los huevos fueron incubados en posición horizontal. A las 48 horas de incubación, se procedió a realizar un orificio en el polo agudo del huevo, del cual se extrajeron $2 \mathrm{~mL}$ de albúmina con ayuda de una pipeta Pasteur con técnica estéril para permitir el descenso de la yema.

Luego se abrió una ventana de $2.5 \mathrm{~cm}$ de ancho por $2 \mathrm{~cm}$ de largo sobre el polo horizontal previamente marcado, la que fue posteriormente sellada con cinta adhesiva, continuando el desarrollo de los huevos en la cámara de cultivo. Al décimo día de incubación, se abrió la ventana horizontal inicial y se depositaron filtros de metilcelulosa sobre la MAC (en la foto 1 se visualiza de forma esquemática la disposición del filtro ya instalado en un embrión a los 10 días de incubación). Se procedió a la instilación de los fármacos a los 5 grupos en dilución de DMSO al 0.1\%, ya que esta concentración no tiene efectos significativos sobre la angiogénesis (Nowak, et al., 2009). 


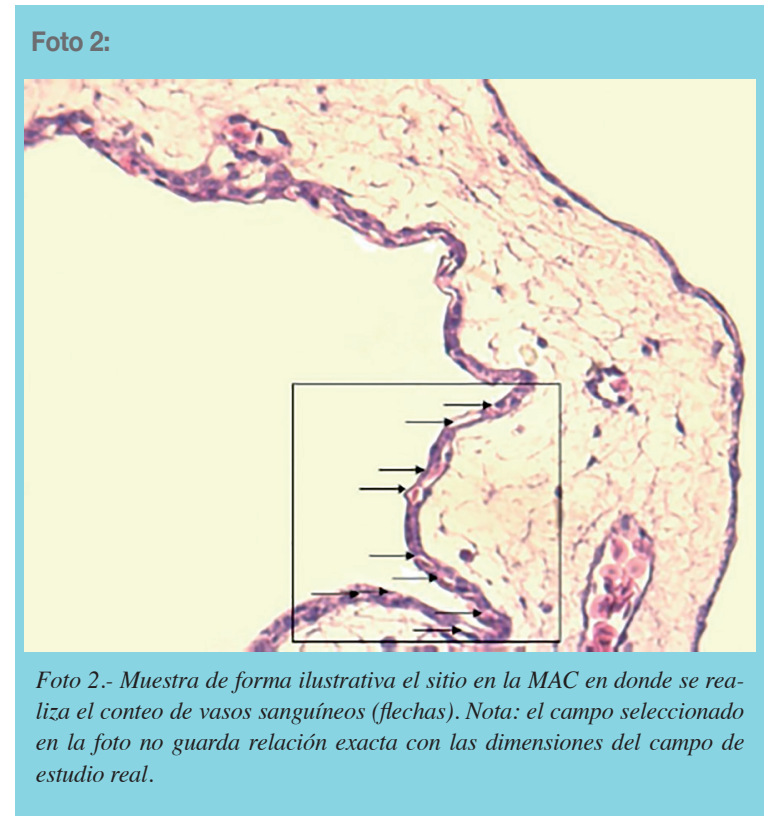

- Grupo Control (N 11): $10 \mu \mathrm{L}$ de DMSO al 0.1\%+m199

- Grupo AAS2 (N 8): $10 \mu \mathrm{L}$ de DMSO al 0.1\% + m199+AAS 2mM

- Grupo AAS5 (N 5): $10 \mu \mathrm{L}$ de DMSO al 0.1\% +m199+ AAS 5.0mM

- Grupo AS2 (N 10): $10 \mu \mathrm{L}$ de DMSO al $0.1 \%+$ m199+AAS 2mM

- Grupo AS5 (N 12): $10 \mu \mathrm{L}$ de DMSO al 0.1\% +m199+ AS $5.0 \mathrm{mM}$

\section{Estudio histológico:}

A las 48 horas post-aplicación de la solución a estudiar se observo una mortalidad de 2 huevos del grupo control, 1 de AAS2mM, 2 AAS5mM y 1 de AS5mM.

Se procedió a la fijación de las muestras de la MAC y mediante deshidratación en alcoholes ascendentes se procedió al montaje con albúmina de los cortes (5 um de espesor) en portaobjetos de vidrio.

Posterior a la obtención de los cortes histológicos de cada MAC se procedió al análisis microscópico de capilares y vasos mayores a una distancia de $9.000 \mu \mathrm{m} 2$ del borde lateral a cada lado del filtro. Para esto se utilizó el microscopio óptico con aumento 400x. El conteo se realizó utilizando un retículo graduado dividido en 10 x10 segmentos que mide en total $90.000 \mu \mathrm{m} 2$, en los cuales se examinaron 10 campos continuos de $900 \mu \mathrm{m} 2 \mathrm{C} / \mathrm{U}$ (foto 2).

El conteo se realizó de forma ciega por 2 examinadores, sin conocer a que grupo correspondían las MAC.

Posteriormente, se procedió a sumar los vasos contabilizados en el campo de estudio de cada extremo del filtro de cada corte de MAC.

Los datos fueron analizados en Graphpad Prism 6.0® con lo que se realizó la prueba ANOVA y posteriormente t de Student entre los vasos sanguíneos contabilizados de los distintos grupos y se procedió a graficar los resultados estandarizando para su interpretación el promedio de cada grupo y su desviación estándar (DE).

\section{Resultados}

Se analizaron un total de 121 cortes histológicos y se estudiaron 242 campos de $9.000 \mu \mathrm{m} 2$

El grupo control en donde la MAC fue expuesta a DMSO al $0.1 \%+$ m199 presentó un promedio de $21.8 \pm 4.2$ vasos sanguíneos. Para el grupo AAS $2 \mathrm{mM}$, el promedio de vasos sanguíneos fue $11.3 \pm 2.6$ y la MAC expuesta a AAS $5 \mathrm{mM}$ tuvo un promedio de $10 \pm 2.9$ vasos sanguíneos. El grupo expuesto a AS $2 \mathrm{mM}$ tuvo un promedio de vaso sanguíneos de $15.6 \pm 3.6$ y el grupo AS $5 \mathrm{mM}$ tuvo un promedio de $12.4 \pm 2.8$ (Tabla 1 ).

Tabla 1.- Número de membranas analizadas de cada grupo, total de cortes examinados, y promedio de vasos sanguíneos con su desviación estándar

\begin{tabular}{cccc} 
& Promedio de vasos & DE & $\begin{array}{c}\text { Cortes } \\
\text { analizados }\end{array}$ \\
\cline { 2 - 4 } CONTROL & 21,8 & 4,2 & 32 \\
AAS2 & 11,3 & 2,6 & 22 \\
AAS5 & 10 & 2,9 & 6 \\
AS2 & 15,6 & 3,6 & 28 \\
AS5 & 12,4 & 2,9 & 33
\end{tabular}

La diferencia entre el número de vasos contabilizados en el grupo control en relación a los demás grupos expuestos a AAS y AS independiente de sus concentraciones fue estadísticamente significativa.

No hubo diferencias significativas entre el número total de vasos contabilizados en la MAC de AAS $5 \mathrm{mM}$ y el grupo AAS 2mM. Para el grupo de MACs expuestas a ácido salicílico, existió diferencia significativa entre ambas concentraciones.

En comparación con el grupo expuesto a aspirina, este sólo obtuvo diferencias significativas en comparación con AS $2 \mathrm{mM}$, sin tener significancia estadística en comparación con AS 5mM (Tabla 2 y Figura 1).

\section{Discusión}

Los resultados de este estudio muestran que el AAS, siendo un agente utilizado ampliamente como antiplaquetario, 


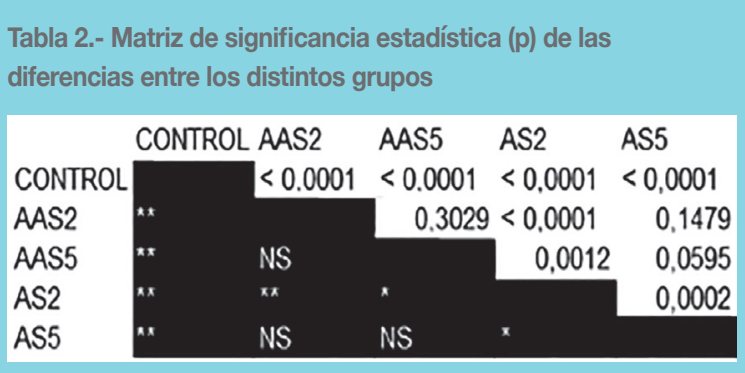

ejerce un efecto antiangiogénico en concentración terapéutica en condiciones de angiogénesis fisiológica.

De manera similar AAS y AS ejercen potentes efectos antiangiogénicos sobre MAC, sin encontrarse diferencias estadísticamente significativas con respecto a las concentraciones estudiadas de AAS, considerando que AAS2 $\mathrm{mM}$ es una concentración terapéutica. Por ello podemos suponer que el efecto antiangiogénico no sería concentración dependiente, como si lo sería aparentemente para el AS, probablemente por un efecto de citotoxicidad directa (Borthwick et al., 2006; Kopp \& Ghosh: 1994; Stark et al., 2001).

La mayor capacidad antiangiogénica del AAS comparado con la del AS radicaría en el efecto que tendría el grupo acetil sobre el metabolismo del ácido araquidónico en la vía de las lipoxinas, siendo estas últimas potentes antiangiogénicos, metabolizadas en los macrófagos (Vane \& Botting., 2003; Gilroy \& Perretti., 2005). Por consiguiente, la ventaja del modelo MAC sobre los modelos "in Vitro" se basa en que en el primero hay participación de macrófagos nativos que interactúan en el tejido en condiciones fisiológicas expuesto a este tipo de agentes.

Lo relevante de este estudio, tomando en cuenta lo mencionado anteriormente, se fundamenta en el uso del AAS en la práctica clínica, principalmente en patología tumoral e isquémica.

Con respecto a la patología isquémica, no se ha postulado un supuesto efecto perjudicial del AAS "dosis dependiente" sobre la angiogénesis miocárdica en la fase de mayor estímulo angiogénico que ocurre durante la primera semana del infarto agudo al miocardio. Es en esta fase que se

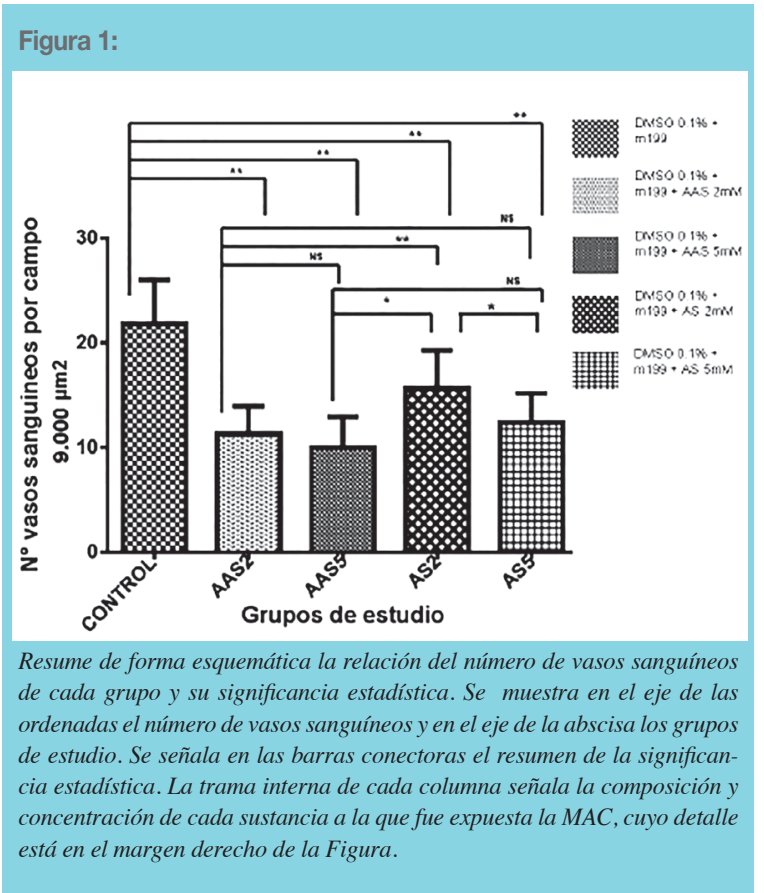

estimula la angiogénesis en brote desde las células endoteliales de los vasos sanguíneos que colindan con el área infartada (May et al., 2008).

Utilizando MAC, el AAS a ha sido estudiado "in vivo" en concentración supra terapéutica $(300 \mathrm{uM})$ en relación a la inhibición de la angiogénesis sobre células tumorales (Sharma, et al. 2001).

Además de la inhibición de VEGF mediante el bloqueo irreversible de la actividad plaquetaria que conlleva un bloqueo del VEGF plaquetario por AAS, este genera una inhibición directa de la expresión tisular de VEGF y disminución del VEGF plasmático circulante (Battinelli et al., 2011, Bachetti., 2013; Cheng et al., 2015).

Por lo tanto, esta investigación genera expectativas para futuros estudios sobre los mecanismos involucrados en el efecto antiangiogénico del AAS involucrados en la modulación de factores proangiogénicos, vías de señalización de supervivencia e inducción de síntesis de moléculas de vías proapoptóticas. 


\section{Bibliografía}

1. BACHETTI T. Adherence junction proteins in angiogenesis: modulation by aspirin and salicylic acid. J Cardiovasc Med. 2013;14:395-6.

2. BATTINELLI EM, MARKENS BA, ITALIANO JE JR. Release of angiogenesis regulatory proteins from platelet alpha granules: modulation of physiologic and pathologic angiogenesis. Blood. 2011;118: 1359-1369.

3. BORTHWICK G M, JOHNSON AS, PARTINGTON M, BURN J, WILSON R, ARTHUR HM. Therapeutic levels of aspirin and salicylate directly inhibit a model of angiogenesis through a Cox-independent mechanism. FASEB J. 2006; 20: 2009-2016.

4. CARMELIET P. Angiogenesis in life, disease and medicine. Nature. 2005; 438: 932-6.

5. CHENG Y, LIN J, LIU J, WANG Y, YAN W, ZHANG M. Decreased vascular endothelial growth factor expression is associated with cell apoptosis in low-dose aspirin-induced gastric mucosal injury. Am J Med Sci. 2015; 349: 110-6.

6. GILROY DW, PERRETTI M. Aspirin and steroids: new mechanistic findings and avenues for drug discovery. Curr Opin Pharmacol. 2005; 5:405-411.

7. KHAIDAKOV M, SON M, JWAHAR L. Adherence junction proteins in angiogenesis: modulation by aspirin and salicylic acid. J Cardiovasc Med. 2012; 13: 187-193.

8. KHAN Q, MEHTA JL. Relevance of platelet-independent effects of aspirin to its salutary effect in atherosclerosis-related events. J Atheroscler Thromb. 2005; 12: 185-190.

9. KOPP E, GHOSH S. Inhibition of NF-kappa B by sodium salicylate and aspirin. Science. 1994; 265, 956-959.

10. MAY D, GILON D, DJONOV V, ITIN A, LAZARUS A, GOR-
DON O, et al. Transgenic system for conditional induction and rescue of chronic myocardial hibernation provides insights into genomic programs of hibernation. Proc Natl Acad Sci U S A. 2008;105: 282-287.

11. NOVOSEL EC, KLEIHANS C, KLUGER PJ. Vascularization is the key challenge in tissue engineering. Advanced drug delivery reviews. 2007; 63, 300-11.

12. NOWAK-SLIWINSKA P, BALLINI JP, VAN DEN BERGH H, WAGNIÈRES G. Vascular effects induced by anti-VEGF agents in the CAM model: effect of the DMSO. Proc. SPIE, 2009; 7380.

13. RIBATTI D, NICO B, VACCAA, PRESTA M. The gelatin sponge-chorioallantoic membrane assay. Nature protocols 2006; 1 : 85-91.

14. RIBATTI D. The Chick Embryo Chorioallantoic membrane in the Study of Angiogenesis and Metastasis. 2010; 1: 13-15 2010; 7: 75-77.

15. SWIFT MR, WEINSTEIN BM. Arterial-venous specification during development. Circ. Res. 2009; 104, 576-588.

16. SHARMA S, GHODDOUSSI M, GAO P, KELLOFF GJ, STEELE VE, KOPELOVICH L. A quantitative angiogenesis model for efficacy testing of chemopreventive agents. Anticancer Res.2001; 21: 3829-37.

17. STARK LA, DIN FV, ZWACKA RM, DUNLOP MG. Aspirin-induced activation of the NF-kappaB signaling pathway: a novel mechanism for aspirin-mediated apoptosis in colon cancer cells. FASEB J. 2001;15: 1273-1275.

18. TOYODA K. Pharmacotherapy for the secondary prevention of stroke. Drugs. 2009; 69: 633-647.

19. VANE JR, BOTTING RM. The mechanism of action of aspirin. Thromb Res. 2003; 110:255-258, 203. 